\title{
Analysis of deformation and microstructure evolution during the hot deformation of titanium alloy
}

\author{
Marcin Kukuryk ${ }^{1, *}$, Jerzy Winczek $^{1}$ \\ ${ }^{1}$ Czestochowa University of Technology, 42-201 Czestochowa, Poland
}

\begin{abstract}
The paper presents theoretical and experimental analysis of deformations and microstructural evolutions in the hot cogging process of Ti-6Al-4V alloy. A three-dimensional thermal - plastic coupled finite element model is employed to study the mechanical and thermal interaction between the forging anvils and the workpiece. To explore the distributions of effective strain, effective stress, mean stress and temperature of the specimens have been systematically studied. Attention has been paid to deformation, temperature, stress and strain inside the specimens and these parameters have been used to determine the evolution of the microstructure in deformed samples during hot cogging process. A comparison of theoretical with experimental results shows that the developed model may be used to accurately predict deformations and microstructural parameters.
\end{abstract}

Keywords: hot forging, titanium alloy, finite element, strain, stress, microstructure evolution

\section{Introduction}

Titanium alloys are well known for industrial applications such as aerospace, automotive, marine and biomedical. Ti-6Al-4V alloy is one of the most important engineering alloys, combining attractive properties such as high strength to density ratio, high strength at relatively high temperatures (up to $550{ }^{\circ} \mathrm{C}$ ), good fatigue properties and excellent heat corrosion resistance [1]. The alloys are manufactured by hot plastic working methods, chiefly by open die forging or die forging. The hot workability of an alloy is normally limited by the generation of various deformation defects that eventually lead to gross failure [2]. The great non-uniformity of deformations in the forging process makes the production of internal-quality titanium forgings much more difficult than imparting the proper shape and geometry to the forgings [3]. In order to maintain a relatively small gradient of properties within the entire forging volume and, at the same time, their required level, cogging process is used in anvils of a purpose-designed shape [4].

The microstructure of titanium alloys is very sensitive to the processing parameters such as forging temperature, strain and strain rate. Especially, microstructure evolution in the hot cogging process has an influence on the mechanical properties of material because the flow

* Corresponding author: kukurykm@itm.pcz.pl

Reviewers: Richard Pastirčák, Eva Tillová 
stress of the material is affected by dynamic recrystallization and grain growth [5]. The determination of temperature distribution within the deformation zone during the titanium alloy forging process is important because of the effect of temperature on the properties and structure of the material being deformed [6].

A finite element method-based model has been developed within the present work, which enables the prediction of the distribution of deformation parameters (strain, stress, temperature) and microstructure evolution during the cogging process of the Ti-6Al-4V alloy in three types of forging anvils. Here, a Johnson-Mehl-Avrami-Kolmogorov type equation describing the relationships between deformation conditions (strain, strain rate and temperature) and DRX volume in $\alpha+\beta$ phase was introduced and fitted for Ti-6Al-4V alloy [7]. The results of the experiment are compared quantitatively and qualitatively with those of simulation.

\section{The material model and experimental procedures}

In this study, the rigid - thermoviscoplastic finite element method is used because it is necessary to solve simultaneously the material - flow problem for a given temperature distribution and heat - transfer problem in the hot cogging process. From the variational principle, the functional, $\phi$ for rigid - viscoplastic material can be written as follows:

$$
\phi=\int_{V} \bar{\sigma} \dot{\varepsilon}_{e} d V+\frac{1}{2} \beta \int_{V} \dot{\varepsilon}_{V}^{2} d V-\int_{S_{T}} T_{i} v_{i} d S
$$

where $\bar{\sigma}$ is the effective stress, $\dot{\varepsilon}_{\mathrm{e}}$ is the effective strain rate, $\dot{\varepsilon}_{\mathrm{v}}$ is the volumetric strain rate, $\mathrm{V}$ is the volume of a deforming workpiece, $\mathrm{T}_{\mathrm{i}}$ is the external force acting on a surface $S_{T}$ and $v_{i}$ velocity, $\beta$ a penalty constant with a large positive value.

The flow stress $(\sigma)$ is a function of strain $(\varepsilon)$, strain rate $(\dot{\varepsilon})$ and temperature $(\mathrm{T})$ (experimental results):

$$
\sigma=f(\varepsilon, \dot{\varepsilon}, T)
$$

The flow curves of the model as per Eq. (2) are consistent with the experimental data in the temperature range of 1073-1273 K, the true strain range of $0.105-0.693$ and the strain rate range of $0.1-1.0 \mathrm{~s}^{-1}$. The flow stress of the Ti-6Al-4V alloy obtained from compression tests is input to the FEM simulation system.

The temperature distribution of the workpiece in the analysis of heat - transfer can be obtained by solving the energy balance equation, expressed by

$$
\rho c_{p} \frac{\partial T}{\partial t}=\nabla^{T}(k \nabla T)+q
$$

where $\mathrm{k}$ is the thermal conductivity coefficient, $\mathrm{T}$ is the temperature, $\rho$ is the density, $\mathrm{c}_{\mathrm{p}}$ is the specific heat, $t$ is the time, $\mathrm{q}=\gamma \bar{\sigma} \dot{\varepsilon}_{\mathrm{e}}$ is the heat generation rate in the deformation body, $\gamma$ is the fraction of mechanical energy converted into heat, and was assumed to be 0.9. The finite element method is then adapted to solve Eqs. (1) and (3) which define the thermo - mechanical state of a body in the cogging process.

The microstructure change taking place by deformation is generally called dynamic recrystallization. It is very difficult to model dynamic recrystallization concurrently during forming. The relationships describing the mechanism of dynamic recrystallization are given by 


$$
\begin{gathered}
\varepsilon_{\mathrm{c}}=8.29 \times 10^{-4} \mathrm{~d}_{0}{ }^{\mathrm{n}_{1}} \dot{\bar{\varepsilon}}^{\mathrm{m}_{1}} \exp \frac{\mathrm{Q}_{1}}{\mathrm{RT}} \\
\mathrm{X}_{\mathrm{DRX}}=1-\exp \left[-\beta_{\mathrm{d}}\left(\frac{\bar{\varepsilon}-\varepsilon_{\mathrm{c}}}{\varepsilon_{0.5}}\right)^{\mathrm{k}_{\mathrm{d}}}\right] \\
\varepsilon_{0,5}=\mathrm{a}_{2} \mathrm{~d}_{0}{ }^{\mathrm{n}_{2}} \dot{\bar{\varepsilon}}^{\mathrm{m}_{2}} \exp \frac{\mathrm{Q}_{2}}{\mathrm{RT}} \\
\mathrm{d}_{\text {DRX }}=\mathrm{a}_{3} \mathrm{~d}_{0}{ }^{\mathrm{h}_{3}} \bar{\varepsilon}^{\mathrm{n}_{3}} \dot{\bar{\varepsilon}}^{\mathrm{m}_{3}} \exp \frac{\mathrm{Q}_{3}}{\mathrm{RT}} \\
\mathrm{d}_{\mathrm{AVG}}=\mathrm{X}_{\text {DRX }} \mathrm{d}_{\text {DRX }}+\left(1-\mathrm{X}_{\text {DRX }}\right) \mathrm{d}_{0}
\end{gathered}
$$

where $\varepsilon_{c}$ is the critical strain at which dynamic recrystallization initiates, $T$ the absolute temperature, $\mathrm{X}_{\mathrm{DRX}}$ the volume fraction of dynamic recrystallization, $\bar{\varepsilon}$ the effective strain, $\dot{\bar{\varepsilon}}$ the effective strain rate, $\varepsilon_{0.5}$ the strain for $50 \%$ recrystallization, $R$ the gas constant, $\mathrm{d}_{0}$ the initial grain size, $d_{D R X}$ the dynamically recrystallized grain size, $\mathrm{a}_{2-3}$ the material data (experimental coefficients), $\mathrm{h}_{1}, \mathrm{n}_{1-3}, \mathrm{~m}_{1-3}$ the material data (experimental coefficients), $\mathrm{Q}_{1-3}$ the activation energies obtained from experiment, $\beta_{\mathrm{d}}$ the material data (experimental coefficient); $\mathrm{k}_{\mathrm{d}}$ the material data (experimental exponent), $\mathrm{d}_{\mathrm{AVG}}$ is the average grain size.

\section{Results and discussion}

The studied Ti-6Al-4V alloy with the composition of 6.20 wt.\% Al, 4.10 wt.\% V, 0.15 wt. $\% \mathrm{Fe}, 0.01$ wt. $\%$ C, 0.005 wt. $\%$ H, 0.005 wt. $\%$ N, 0.14 wt.\% O and the balance of Ti. The $\beta$ transus temperature was measured to be approximately $1250 \mathrm{~K}$ by thermal dilatation method. The main input temperature - dependent parameters for simulations were flow stress, thermal conductivity, and heat capacity. The flow stress was represented as a function of strain, strain rate and temperature. The variations of flow stress with temperature were generated for a range of temperatures $(1073-1273 \mathrm{~K}$ with a step of 50 $\mathrm{K})$ and strain rate conditions $\left(0.05,0.10,0.50\right.$, and $\left.1.0 \mathrm{~s}^{-1}\right)$ by compression tests in a MAEKAWA - Japan plastometer. The temperature of the specimens was monitored using a chromel - alumel thermocouple. The total deformation, as calculated from the specimen height change, was $0.105-0.693$. The obtained results allowed the determination of the flow stress values, as dependent on the actual strain, strain rate and temperature, which were input to the program with the aim of evaluating the best rheological model for the material being deformed. The flow curves for lowest $\left(0.10 \mathrm{~s}^{-1)}\right.$ and highest $\left(1.0 \mathrm{~s}^{-1}\right)$ strain rate conditions are shown in Fig. 1 for brevity. It can be clearly seen that the flow stress increases to a maximum during high temperature deformation. After attaining a peak value, the stress decreases until it reaches a steady state. This is an indication that the material softens by dynamic recrystallization.

For the titanium alloy under examination and for the anvil material X37CrMoV51, the thermal characteristics, such as density, specific heat and thermal conductivity, were assumed based on experimental data and were input as functions of temperature. The initial anvil temperature was taken as $300^{\circ} \mathrm{C}$, i.e. the anvil heating temperature in industrial conditions. Some more important values of the determined coefficients occurring in formulas (4)-(8) are as follows: 


$$
\begin{aligned}
& a_{2}=0.0011, n_{1}=0.40, m_{1}=0.017, Q_{1}=41700, \beta_{d}=0.6903, a_{2}=0.0011, n_{2}=0.25, m_{2}=0.05, \\
& Q_{2}=24430, a_{3}=1600, h_{3}=0.70, n_{3}=0.80, Q_{3}=-52235
\end{aligned}
$$
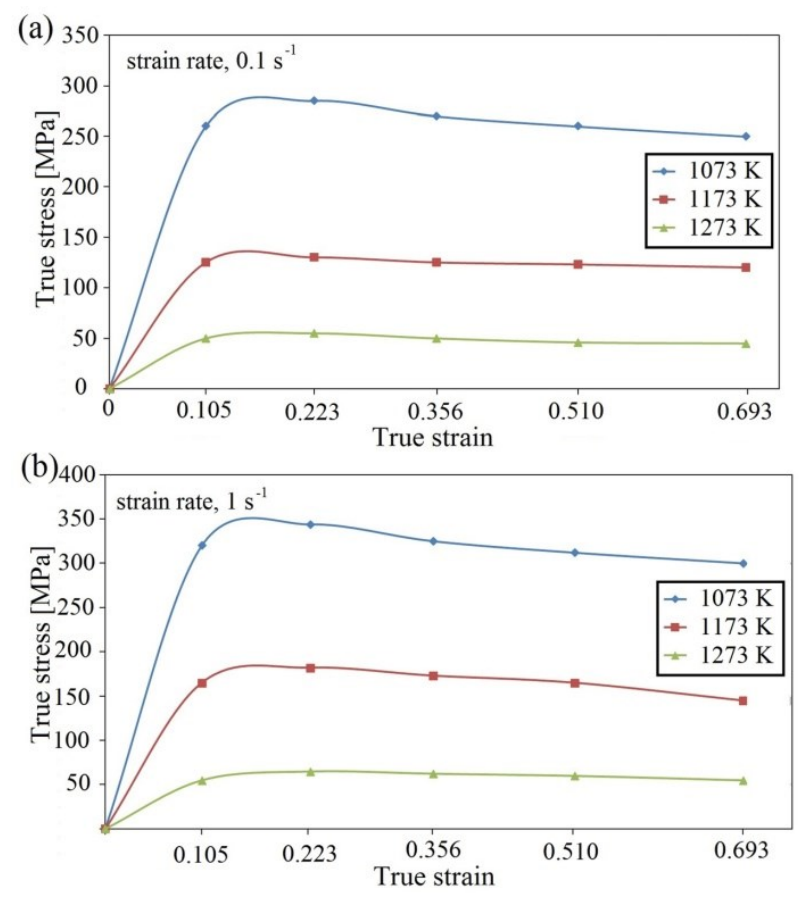

Fig. 1. Variation of flow stress for the Ti-6Al-4V alloy as a function of true strain and temperature, for different strain rate values, i.e.: (a) $-\dot{\varepsilon}=0.1 \mathrm{~s}^{-1}$ and (b) $-\dot{\varepsilon}=1 \mathrm{~s}^{-1}$

In numerical computations and in experimental tests, $\varnothing 80 \mathrm{~mm}$-diameter and $200 \mathrm{~mm}$ long stock of the Ti-6Al-4V alloy was taken. The cogging process consists of many partial, interrelated reductions made in a specific order. Assuming the cyclical nature and repeatability of the phenomena, the study of the stress and strain state can be limited to the examination of one engineering pass (two consecutive reductions with the simultaneous rotation of the material by $90^{\circ}$ ), which will considerably simplify the forging process. Forging was carried out in two consecutive reductions with tilting the workpiece by an angle of $90^{\circ}$, while retaining a constant value of true reduction of $\varepsilon_{\mathrm{h}}=0.35$ and a constant relative feed of $1_{\mathrm{w}}=0.75$. These samples have an initial grain size $\left(d_{o}\right)$ of around $50 \mu \mathrm{m}$ before deformation. The cogging process was conducted in different shape of the anvils: with oblique working surfaces, combined (bottom anvil is V-shaped) and asymmetrical V shaped with an angle of $\alpha_{1}=105^{\circ}$ and $\alpha_{2}=135^{\circ}$, as shown in Fig. 2. The tests carried out enabled the determination of the local values describing the strain state, the stress state, the temperature distribution, and microstructural evolution during cogging process of the Ti$6 \mathrm{Al}-4 \mathrm{~V}$ alloy. 
(a)

(b)
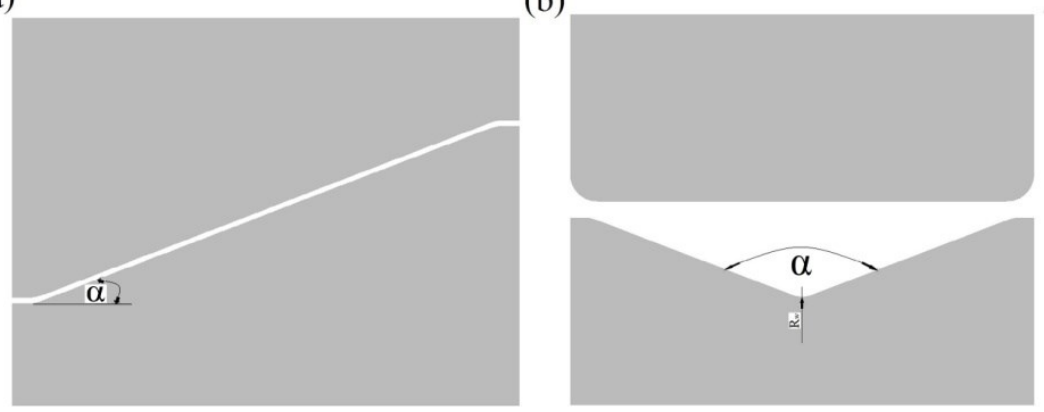

(c)

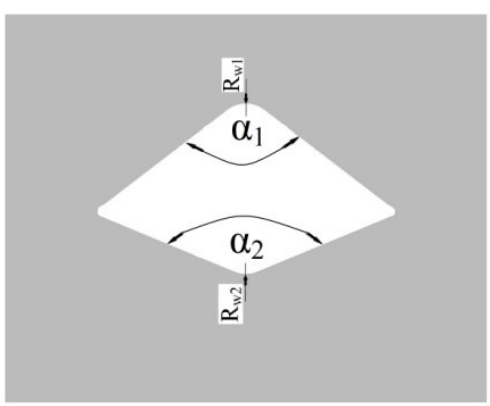

Fig. 2. Shape of anvils: (a) - with oblique working surfaces $\left(\alpha=30^{\circ}\right)$, (b) - combined anvils (bottom anvil is V-shape) with an angle of $\alpha=135^{\circ}\left(\mathrm{R}_{\mathrm{w}}=0.35 \mathrm{D}_{0} ; D_{0}=80 \mathrm{~mm}\right)$, (c) - asymmetrical V - shaped with an angle of $\alpha_{1}=105^{\circ}$ and $\alpha_{2}=135^{\circ}\left(R_{w 1}=R_{w 2}=0.35 D_{0}\right)$

In the case of the elongation of a material having a circular section on anvils with oblique working surfaces (Fig. 3a), the non-homogeneousness of deformations is comparatively large. In the presented distribution of the effective strain following the one engineering pass $\left(\varepsilon_{\mathrm{h}}=0.70\right)$, it is possible to differentiate a number of characteristic zones. Area situated adjacently to surface of anvils is experiencing significantly small deformations $\left(\varepsilon_{\mathrm{i}} / \varepsilon_{\mathrm{h}}=0.48-0.67\right)$. The largest deformation occurred in the central part of a forging, whereas the values of the effective strain $\varepsilon_{i}$ significantly exceeded the value of true strain $\varepsilon_{\mathrm{h}}\left(\varepsilon_{\mathrm{i}} / \varepsilon_{\mathrm{h}}=1.46\right)$, the lateral zones of a forging are the area of intermediate deformations $\left(\varepsilon_{\mathrm{i}} / \varepsilon_{\mathrm{h}}=0.87-1.07\right)$. This part of the material of a forging, in addition to that, is subjected to the effect of tensile stresses for the part of non-deformed external zones, and also for the part of a strongly deformed center (Fig. 3b). The process of forging was accompanied by the stable distribution of temperature in the central part of a forging resulting from the emission of the heat of plastic deformations, and the contact point of a hot metal and cool tools resulted in the formation of the significant gradients of temperature in the region adjacent to the contact surfaces $\left(\Delta \mathrm{T}=80-180^{\circ} \mathrm{C}\right)$. Measurements with the application of a thermovision camera of the temperature of the surface of a forging following deformation demonstrated the compatibility of this temperature with the calculated one (differences $\pm 10{ }^{\circ} \mathrm{C}$ ). 
(a)

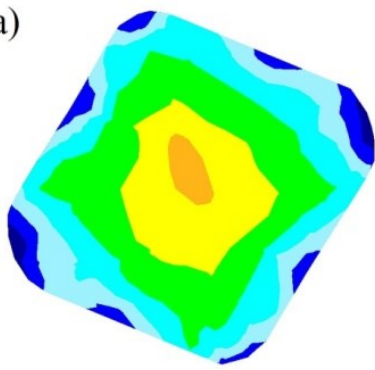

(b)

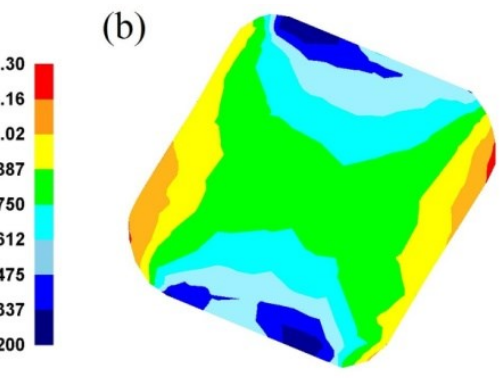

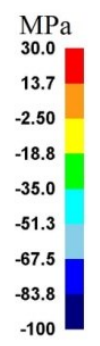

(c)
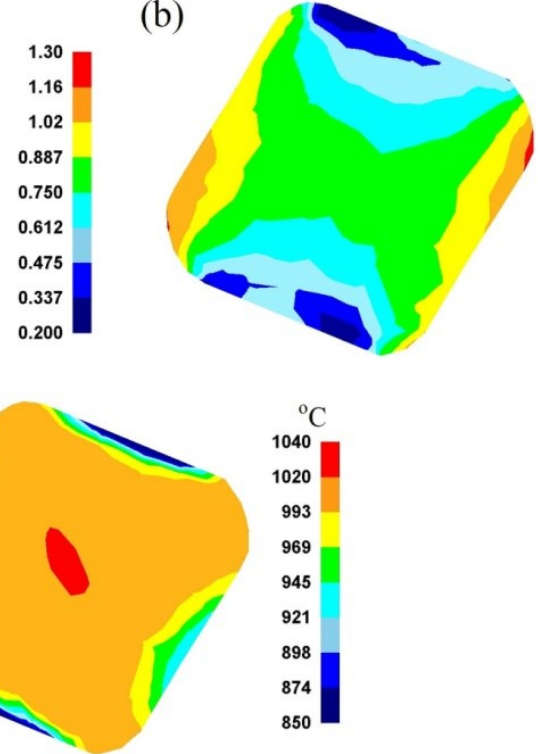

Fig. 3. Distribution of effective strain (a), mean stress (b) and temperature (c) on the cross-sectional surface of the specimens after cogging process in anvils with oblique working surfaces.

$$
\text { True strain } \varepsilon_{\mathrm{h}}=0.70
$$

In Fig. 4, the distribution of the effective strain and mean stress following the cogging process of samples made of the titanium alloy (Ti-6Al-4V) on combined anvils with an angle of $\alpha=135^{\circ}$, following the one engineering pass $\left(\varepsilon_{\mathrm{h}}=0.70\right)$, were presented. The applied anvils demonstrated a similar distribution of the effective strain and mean stress in the cogging process of the titanium alloy being investigated.

(a)

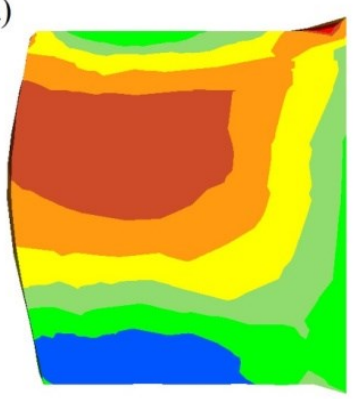

(b)

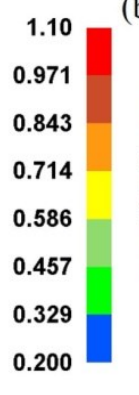

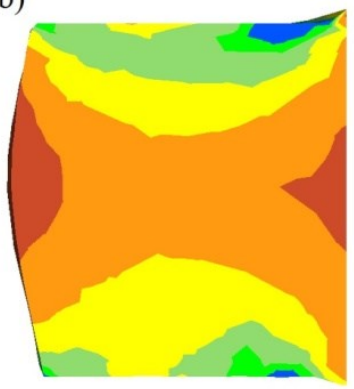

$\mathrm{MPa}$

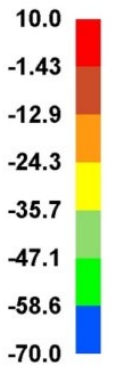

Fig. 4. Distribution of effective strain (a) and mean stress (b) in the longitudinal-section of the specimens after cogging process in combined anvils with $\alpha=135^{\circ}$. True strain $\varepsilon_{\mathrm{h}}=0.70$

In Fig. 5, the distribution of effective strain, mean stress and temperature on the crosssectional surface of the specimens after cogging process in asymmetrical $\mathrm{V}$ - shaped anvils with an angle of $\alpha_{1}=105^{\circ}$ and $\alpha_{2}=135^{\circ}$, following the one engineering pass $\left(\varepsilon_{\mathrm{h}}=0.70\right)$, were presented. The anvils applied in investigations demonstrated a positive effect on the value, and on the homogeneousness, of the distribution of the effective strain, and also resulted in the absence of tensile stress in the central part of the deformation basin (Fig. 5a and 5b). On the larger part of the surface of the transverse section of a sample, the effective strain significantly exceeded the value of the true strain $\left(\varepsilon_{\mathrm{i}} / \varepsilon_{\mathrm{h}}=1.52-1.94\right)$, which had a 
positive effect on the re-forging of it. The lateral zones of a forging were the collections of the smaller values of the effective strain: $\varepsilon_{\mathrm{i}} / \varepsilon_{\mathrm{h}}=1.12$. The presented distribution of mean stress (Fig. 5b) indicates the possibility of the appearance of tensile stress only on a very small area situated in the lateral zones of a forging. The process of forging was accompanied by the stable distribution of temperature in the central part of a forging resulting from the emission of the heat of plastic deformations, and a comparatively large surface of the contact point between the deformed metal and the cool tools resulted in the formation of the significant gradients of temperature $\left(\Delta T=150-200{ }^{\circ} \mathrm{C}\right)$.

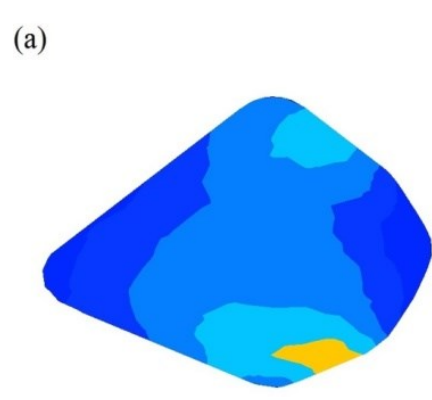

(a)

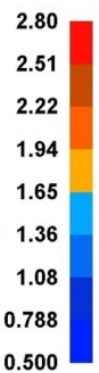

(b)

(c)

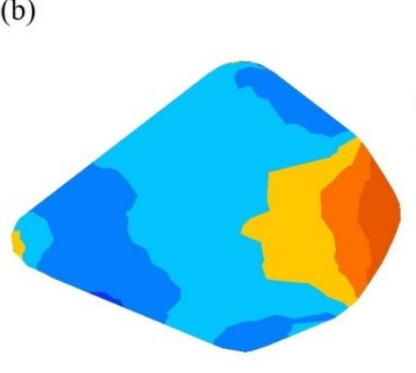

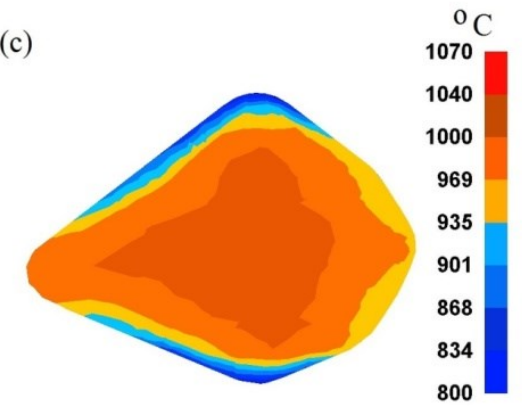

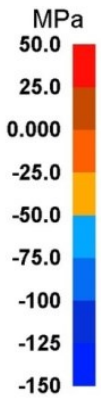

$-150$

Fig. 5. Distribution of effective strain (a), mean stress (b) and temperature (c) on the cross-sectional surface of the specimens after cogging process in asymmetrical $\mathrm{V}$ - shaped anvils with an angle of $\alpha_{1}=105^{\circ}$ and $\alpha_{2}=135^{\circ}$. True strain $\varepsilon_{\mathrm{h}}=0.70$

The distribution of the dynamically recrystallized volume fraction and average grain size on the cross - sectional surface of the specimens after cogging process (after the one engineering pass) in anvils with oblique working surfaces was presented in Fig. 6. The process of deformations was progressing in an uneven manner across the sample, and that brought the fruit of the presence of zones having the different degrees of recrystallizing and different average grain size. The dynamically recrystallized volume fraction reached its maximum value in the central point of a forging, and it amounted to $61.2 \%$ (Fig. 6a). The distribution of the average grain size on the cross - sectional surface of the specimens of was not even. The smallest grain size was obtained for the central area of a forging, which amounted to $24.1 \mu \mathrm{m}$ (Fig. 6b). On the surface of contact between a deformed metal and the anvils, the significantly larger average grain size $(44.8 \mu \mathrm{m})$ was obtained. 
(a)

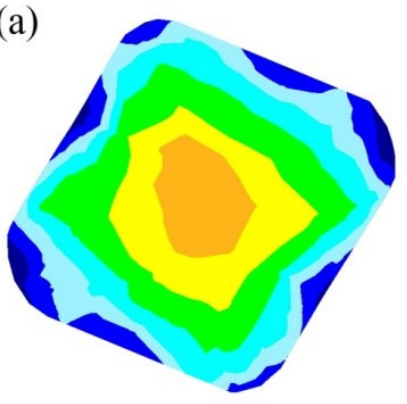

(b)

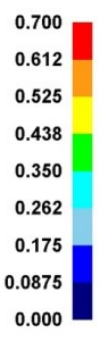

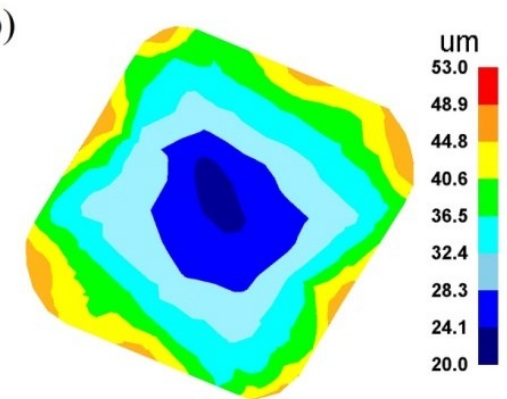

Fig. 6. Distribution of the dynamically recrystallized volume fraction (a) and average grain size (b) on the cross - sectional surface of the specimens after cogging process in anvils with oblique working surfaces. True strain $\varepsilon_{h}=0.70$

For combined anvils, the dynamically recrystallized volume fraction reached its maximum value in the central point of a forging, and it amounted to $85.7 \%$ (Fig. 7a). The smallest grain size was obtained for the central area of a forging, and it amounted to 16.3 $\mu \mathrm{m}$ (Fig. 7b). On the surface of contact between the deformed metal and the anvils, a significantly larger average grain size $(41.4-47.7 \mu \mathrm{m})$ was obtained.

(a)

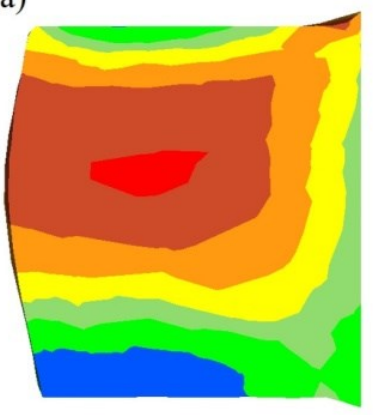

(b)

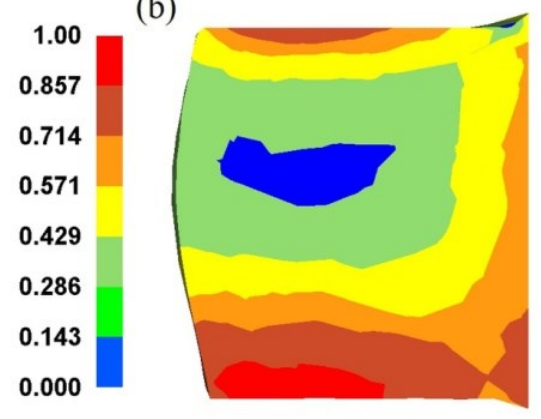

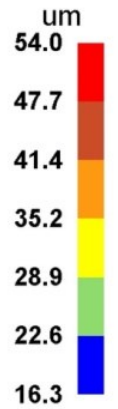

16.3

Fig. 7. Distribution of the dynamically recrystallized volume fraction (a) and average grain size (b) in the longitudinal section of the specimens after cogging process in combined anvils with $\alpha=135^{\circ}$.

True strain $\varepsilon_{\mathrm{h}}=0.70$

The distribution of the dynamically recrystallized volume fraction, and also the distribution of the average grain size on the cross - sectional surface of the specimens after cogging process (after the one engineering pass) on asymmetrical $\mathrm{V}$-shaped anvils with the angle of $\alpha_{1}=105^{\circ}$ and $\alpha_{2}=135^{\circ}$ was presented in Fig. 8. The microstructure which was obtained was to a large degree recrystallized and homogeneous on a dominant part of the section of a sample. The dynamical recrystallization was commencing on the large part of the area subjected to the largest deformation situated in the axial zone of a forging, where the dynamically recrystallized volume fraction reached its maximum value. For the lateral zones of a forging, the dynamically recrystallized volume fraction reached smaller values, and it amounted to $62.5-75.0 \%$ (Fig. 8a). The distribution of the average grain size on the cross - sectional surface of the specimens was more even in comparison with the distributions on the previously-researched anvils. The intensive re-forging of the axial zone of a forging in the asymmetrical $\mathrm{V}$-shaped anvils resulted in the formation, in this area, of 
the most grinded structure, which amounted to $11.3-15.0 \mu \mathrm{m}$ (Fig. 8b). For the lateral zone of a forging, the average grain size was larger, and amounted to $22.5-26.3 \mu \mathrm{m}$.

The conducted comparative analysis gives rise to the conclusion that the largest effect of the grinding of a grain size in the axial zone of a forging in the course of the forging of the Ti-6Al-4V titanium alloy was obtained on asymmetrical $\mathrm{V}$-shaped anvils with an angle of $\alpha_{1}=105^{\circ}$ and $\alpha_{2}=135^{\circ}$, in the case of which, simultaneously with increase in true strain, there increased the effective strain, which was accompanied by a significant reduction in the grain size. Introduction, in the initial phase of cogging process on asymmetrical Vshaped anvils, will make it possible to reach even plastic processing in the volume of a forging, and also obtaining the homogeneous and finely-crystallite microstructure. The final deformation may be conducted in combined anvils, or in the ones with oblique working surfaces.

(a)

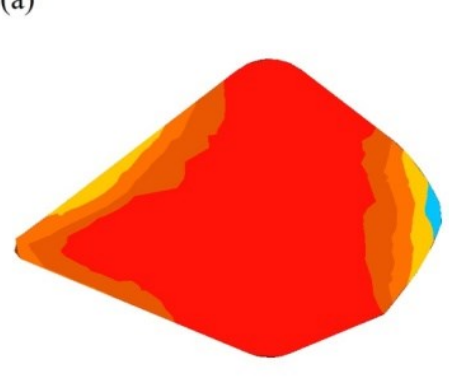

(b)

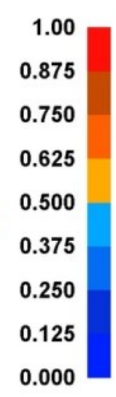

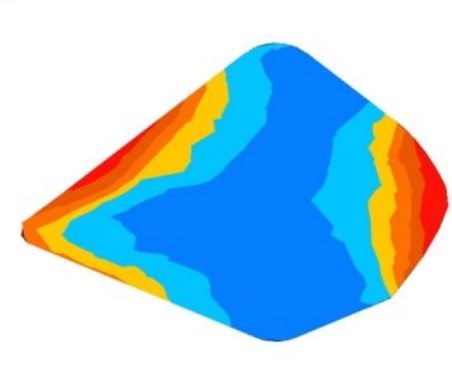

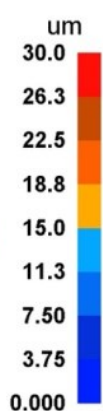

Fig. 8. Distribution of the dynamically recrystallized volume fraction (a) and average grain size (b) on the cross-sectional surface of the specimens after cogging process in asymmetrical $\mathrm{V}$ - shaped anvils with an angle of $\alpha_{1}=105^{\circ}$ and $\alpha_{2}=135^{\circ}$. True strain $\varepsilon_{\mathrm{h}}=0.70$

The experimental confirmation of the results of numerical research into the distribution of the effective strain on asymmetrical V-shaped anvils with the angle of $\alpha_{1}=105^{\circ}$ and $\alpha_{2}=$ $135^{\circ}$ was presented in Fig. 9. The state of deformations was determined with the application of the coordinate grid method. The experimental research was conducted on cylindrical samples having the following dimensions: $\phi 80 \times 150 \mathrm{~mm}$ and made of the Ti-6Al-4V alloy, deformed on a hydraulic press having the pressure amounting to $2.500 \mathrm{kN}$.

(a)

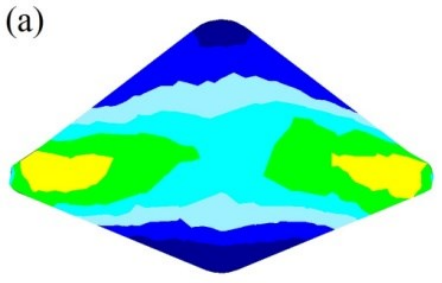

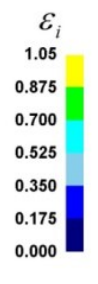

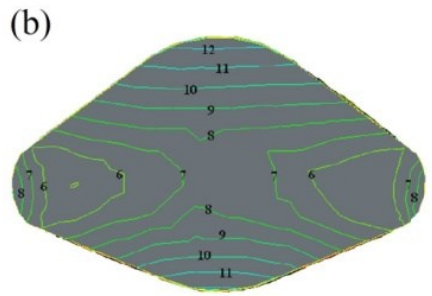

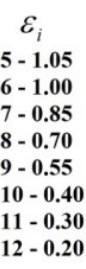

Fig. 9. Comparison of the theoretical (a) and experimental (b) distribution effective strain $\left(\varepsilon_{\mathrm{i}}\right)$ on the cross-sectional surface of the specimens after cogging process in asymmetrical $\mathrm{V}$ - shaped anvils with an angle of $\alpha_{1}=105^{\circ}$ and $\alpha_{2}=135^{\circ}$. Relative feed $l_{w}=0.75$, true strain $\varepsilon_{\mathrm{h}}=0.35$

In order to determine the constituents of the tensor of finite Cauchy deformations, the displacement field was approximated with the application of the two-parameter polynomials of degree six; in the case of that, the coefficients were determined with the application of the method of least squares, in the case of taking under consideration the 
condition of constant volume (with the application of the method of Lagrange multiplier). The conducted comparative analysis of the effective strain from the numerical calculations and those obtained by means of experimental method demonstrated a comparatively good compatibility of them.

The experimental measurement of temperature was performed on the surface of deformed samples made of the Ti-6Al-4V alloy with the application of a thermovision camera. The compatibility of the calculated and measured temperatures obtained for the surface layer (Fig. 10a) makes it possible to presume that the simulated distribution of temperatures on the section of a forged band are compatible with the actual ones.
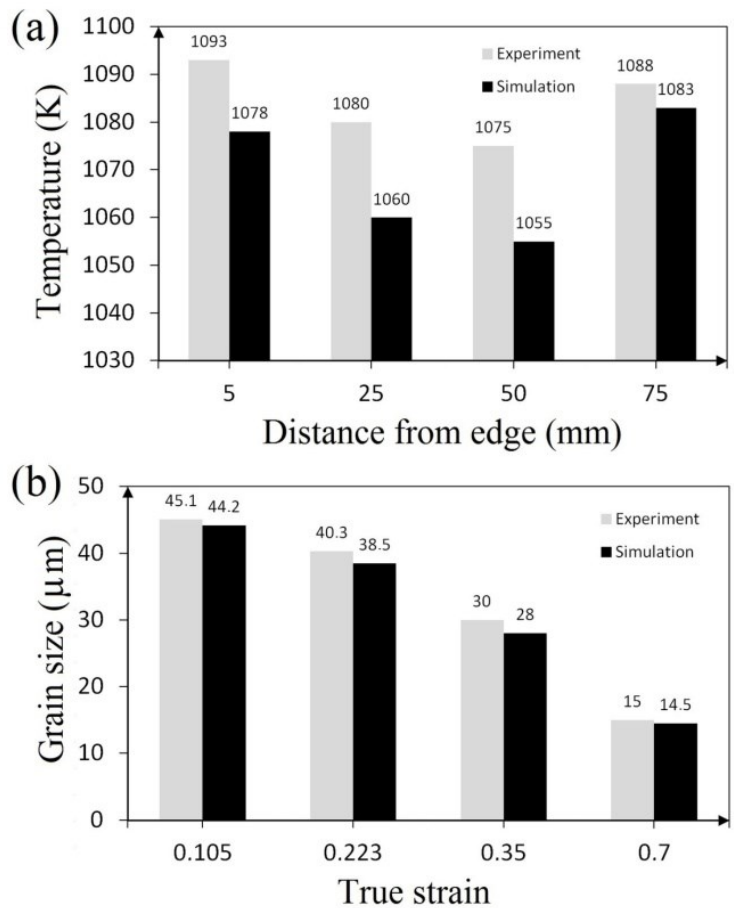

Fig. 10. Comparisons between the experimental and simulated results after cogging process in asymmetrical $\mathrm{V}$-shaped anvils with an angle of $\alpha_{1}=105^{\circ}$ and $\alpha_{2}=135^{\circ}$ : (a) temperature in the longitudinal section of the specimens $\left(l_{w}=0.75 ; \varepsilon_{\mathrm{h}}=0.70\right)$, (b) average grain sizes in the centre of the specimens deformed at different true strain

The experimental measurement of the average grain size was performed for a number of representative samples made of the Ti-6Al-4V alloy, which were re-forged on asymmetrical V-shaped anvils with an angle of $\alpha_{1}=105^{\circ}$ and $\alpha_{2}=135^{\circ}$, with the application of the following parameters of deformations: $\dot{\varepsilon}=0.25 \mathrm{~s}^{-1} ; T_{\mathrm{o}}=1173 \mathrm{~K} ; \varepsilon_{\mathrm{h}}=0.105-0.693$. On the selected samples, a number of measurements of the average grain size by means of the comparative method and in accordance with the standard: PN-EN ISO 643:2013-06 (Fig. 10b) were performed. 


\section{Conclusions}

By means of the selection of the appropriate shape and geometry of the working surface of anvils, and also rational technical parameters, it is possible to exert a significant influence upon: the situation of the maximum values of the effective strain, the value of the nonhomogeneousness of the distribution of deformations, and also upon reduction in the effect of tensile stress in the deformation basin. The process of forging on asymmetrical V-shaped anvils was accompanied by a small gradient of temperature in central part of the deformation basin contributing to increase in the homogeneousness of deformations, and that brought the fruit of obtaining a microstructure recrystallized to a large degree, and homogeneous. In the central part of the deformation basin, the largest reduction the average grain size was obtained (11.3-15.0 $\mu \mathrm{m})$. In contact zones and also for the lateral surfaces of a forging, the average grain size was significantly larger, whereas it was reducing approximately two times. In the course of the cogging process on asymmetrical $\mathrm{V}$-shaped anvils with an angle of $\alpha_{1}=105^{\circ}$ and $\alpha_{2}=135^{\circ}$, as a result of the intensive re-forging of the central areas of a forging, a twice smaller the average grain size was obtained in them in comparison with the average grain size was obtained in the course of forging on the remaining researched anvils.

The application in the initial phase of the cogging process on asymmetrical V-shaped anvils will make it possible to reach the large values of the effective strain in the volume of the deformed material, with the simultaneous maintenance of the high homogeneousness of the distribution of it. The final operation of the cogging process may be conducted in oblique anvils, or in the combined ones. The combination of different anvils in the process of the cogging process of the two-phase alloys of titanium provides the possibilities of summary use of their particular assets.

\section{References}

1 N. Kotkunde, A.D. Deole, A.K. Gupta, S.K. Singh, Comparative study of constitutive modeling for Ti-6Al-4V alloy at low strain rates and elevated temperatures. Materials and Design 55, 999-1005 (2014)

2 T.R. Prabhu, Simulations and experiments of the nonisothermal forging process of a Ti- 6Al-4V impeller. Journal of Materials Engineering and Performance 25 (9), 36273637 (2016)

3 S. Luo, D. Zhu, L. Hua, D. Qian, S. Yan, F. Yu, Effects of process parameters on deformation and temperature uniformity of forged Ti-6Al-4V turbine blade. Journal of Materials Engineering and Performance 25 (11), 4824- 4836 (2016)

4 S.K. Choi, M.S. Chun, C.J. Van -Tyne, Y.H. Moon, Optimization of open die forging of round shapes using FEM analysis. Journal of Materials Processing Technology 172, 88-95 (2006)

5 RG. Guan, Y.T. Je, Z.Y. Zhao, Ch.S. Lee, Effect of microstructure on deformation behavior of Ti-6Al-4V alloy during compressing process. Materials and Design 36, 796-803 (2012)

6 G. Chen, Ch. Ren, X. Qin, J. Li, Temperature dependent work hardening in Ti-6Al-4V alloy over large temperature and strain rate ranges: experiments and constitutive modeling. Materials and Design 83, 598-610 (2015)

7 G.Z. Quan, G.Ch. Luo, J.T. Liang, D.S. Wu, A. Mao, Q. Liu, Modelling for the dynamic recrystallization evolution of Ti-6Al-4V alloy in two-phase temperature range and a wide strain rate range. Computational Materials Science 97, 136-147 (2015) 